\title{
AN EXAMPLE ON STRONG UNICITY CONSTANTS IN TRIGONOMETRIC APPROXIMATION
}

\author{
K. Y. CHAN, Y. M. CHEN, M. C. LIU AND S. M. NG
}

\begin{abstract}
In this paper an explicit example is constructed to illustrate some interesting properties of the strong unicity constant in the case of approximations by trigonometric polynomials.
\end{abstract}

1. Introduction. Let $C(I)$ be the space of all real-valued continuous functions defined on the closed and bounded interval $I$, with the uniform norm $\|f\|=$ $\max _{x \in I}|f(x)|$ for $f \in C(I)$. Let $G \subset C(I)$ be any finite-dimensional Haar subspace $[1$, p. 74]. It is known [1, pp. 75, 80] that, for each $f \in C(I)$, there exists a unique $g^{*} \in G$, called the element of best approximation to $f$ from $G$, such that

$$
\left\|g^{*}-f\right\| \leqslant\|g-f\|
$$

for any $g \in G$, and that there exists a positive constant $K$ depending on $f$ and $G$ but not on $g$ such that

$$
\left\|g-g^{*}\right\|<K\left(\|f-g\|-\left\|f-g^{*}\right\|\right)
$$

for any $g \in G$. The least constant $K$ satisfying (1.1) is called the strong unicity constant of $f$ for $G$. Two particularly interesting Haar subspaces are the set of all algebraic polynomials and the set of all trigonometric polynomials of degree at most $n$. We denote the two sets by $\Pi_{n}$ and $\Pi_{n}^{T}$ respectively and the corresponding strong unicity constants of $f$ by $M_{n}(f)$ and $M_{n}^{T}(f)$. Our interest in this paper centers on the problem of approximating the functions in $C(I)$ by trigonometric polynomials in $\Pi_{n}^{T}$, where $I=[0,2 \pi]$.

S. J. Poreda [5, Theorem 2] first pointed out that there exists a $f \in C(I)$ for which the sequence $\left\{M_{n}(f)\right\}_{n-1}^{\infty}$ is unbounded. He also posed [5, p. 170] the problem of characterizing those functions $f \in C(I)$ for which the above sequence is bounded. M. S. Henry and J. A. Roulier further studied the problem and made the conjecture $[3$, p. 94$]$ that the sequence is bounded only for $f \in \Pi_{n}$. These problems are still unsettled. Later D. Schmidt [6, Theorem 6] showed the existence of a $f \in C([-1,1])$ such that

$$
\underset{n \rightarrow \infty}{\lim _{n}} M_{n}(f)=1 \text { and } \varlimsup_{n \rightarrow \infty} M_{n}(f)=+\infty .
$$

The structure of Schmidt's example is somewhat similar to that of Poreda's [5, p. 168]. Both authors made use of a sequence of special algebraic polynomials based on a theorem due to W. Wolibner [7].

Received by the editors July 22, 1980.

1980 Mathematics Subject Classification. Primary 42A10; Secondary 41A10.

Key words and phrases. Strong unicity constant, trigonometric polynomials, best approximation.

(C) 1982 American Mathematical Society 0002-9939/82/0000-0018/\$02.50 
Since many results in Approximation Theory occur in pairs, one for algebraic polynomials and a similar one for trigonometric polynomials, we find it natural also to conjecture that $\left\{M_{n}^{T}(f)\right\}_{n=1}^{\infty}$ is bounded only for $f \in \Pi_{n}^{T}$ and to expect results similar to (1.2) for trigonometric polynomials in $\Pi_{n}^{T}$. Moreover such results may indicate whether it is profitable to look for general theorems that hold for any Haar subspace. ${ }^{1}$ For example, let $G_{1} \subset G_{2} \subset \cdots \subset G_{n} \subset \ldots$ be any sequence of finite-dimensional Haar subspaces of $C(I)$. We may ask the question whether the sequence of strong unicity constants of $f \in C(I)$ for the Haar subspaces $\left\{G_{n}\right\}_{n=1}^{\infty}$ is bounded only for $f$ in some $G_{n}$ or not. However, Wolibner's theorem [7] and hence the methods which all previous writers $[5$, p. 168], $[6$, p. 221], $[3$, p. 91] used to show the unboundedness of $\left\{M_{n}(f)\right\}_{n=1}^{\infty}$ cannot readily be extended from algebraic polynomials to trigonometric polynomials. In this paper, we have succeeded in extending some of the results for algebraic polynomials to trigonometric polynomials. Without using Wolibner's theorem, we are going to construct an explicit example by which we shall be able to extend Schmidt's result to $\Pi_{n}^{T}$ and to obtain a result similar to (1.2) for a function which has continuous derivatives up to any given finite order. Our result also shows that the strong unicity constants $M_{n}^{T}(f)$ for two adjacent values of $n$ may fluctuate violently between the supremum and infimum values $+\infty$ and 1 as $n \rightarrow \infty$. Furthermore, let $C_{2 \pi}$ be the space of real-valued $2 \pi$-periodic continuous functions defined on the real line (with the uniform norm), and let the set be denoted by $C_{2 \pi}[0,2 \pi]$ when the functions in $C_{2 \pi}$ are restricted to the closed interval $[0,2 \pi]$. We have $C_{2 \pi}[0,2 \pi] \subset C([0,2 \pi])$. Since our results remain valid when we consider only functions $f \in C_{2 \pi}[0,2 \pi]$, the results in this paper also hold when $C_{2 \pi}$ is substituted for $C([0,2 \pi])$.

2. Condition for $\lim _{n \rightarrow \infty} M_{n}^{T}(f)=1$. For each $f \in C([0,2 \pi])$, let $T_{n}(f) \in \Pi_{n}^{T}$ be the trigonometric polynomial of best approximation to $f$ and let

$$
E_{n}(f)=\left\{x \in[0,2 \pi]:\left|\left(f-T_{n}(f)\right)(x)\right|=\left\|f-T_{n}(f)\right\|\right\} .
$$

A set $A_{n}(f)=\left\{x_{i} \in E_{n}(f): i=0,1, \ldots, s ; x_{0}<x_{1}<\cdots<x_{s}\right\}$ is called an alternating system if

(i) $s \geqslant 2 n+1$,

(ii) $\operatorname{sgn}\left(f-T_{n}(f)\right)\left(x_{i}\right)=-\operatorname{sgn}\left(f-T_{n}(f)\right)\left(x_{i+1}\right), i=0,1, \ldots, s-1$.

THEOREM 1. Suppose that $f \in C([0,2 \pi])$ is not a trigonometric polynomial. Let $\left\{\varepsilon_{i}\right\}_{i=1}^{\infty}$ be a sequence of positive numbers such that $\lim _{i \rightarrow \infty} \varepsilon_{i}=0$. If there exists $a$ strictly increasing sequence $\left\{n_{i}\right\}_{i=1}^{\infty}$ of positive integers such that corresponding to each $n_{i}$ there is an $A_{n_{i}}(f)$ satisfying

$$
\max _{x_{j}, x_{j+1} \in \mathbb{Q}_{n_{i}}}\left|x_{j}-x_{j+1}\right| \leqslant \varepsilon_{i} / n_{i},
$$

where $\mathbb{Q}_{n_{i}}=A_{n_{i}}(f) \cup\{2 \pi, 0\}$ and $x_{j}, x_{j+1}$ are two adjacent points in $\mathbb{Q}_{n_{i}}$, then

$$
\lim _{i \rightarrow \infty} M_{n_{i}}^{T}(f)=1 \text {. }
$$

\footnotetext{
${ }^{1}$ We are grateful to the referee for pointing out this point to us.
} 
Proof. Let

$$
S_{n_{i}}=\left\{P \in \Pi_{n_{i}}^{T}:\|P\|=1\right\} \text {. }
$$

For any $P \in S_{n_{i}}$, there is a $y \in[0,2 \pi]$ such that $|P(y)|=1$. By (2.2) there are two adjacent points $x_{k}, x_{k+1} \in A_{n_{i}}(f)$ such that

$$
\max \left(\left|x_{k}-y\right|,\left|x_{k+1}-y\right|\right)<2 \varepsilon_{i} / n_{i} \text {. }
$$

Since $f-T_{n_{i}}(f)$ has different signs at $x_{k}$ and $x_{k+1}$, we may suppose, without loss of generality, that

$$
\operatorname{sgn} P(y)=\operatorname{sgn}\left(f-T_{n_{i}}(f)\right)\left(x_{k}\right)
$$

By the mean value theorem

$$
\left|P(y)-P\left(x_{k}\right)\right|=P^{\prime}(\eta)\left|y-x_{k}\right|
$$

for some $\eta$ lying between $y$ and $x_{k}$, and by Bernstein's inequality [1, p. 91],

$$
\left|P^{\prime}(\eta)\right| \leqslant n_{i}\|P\| \text {. }
$$

Hence from (2.3) and (2.4) we have

$$
\left|P(y)-P\left(x_{k}\right)\right| \leqslant n_{i}\|P\| 2 \varepsilon_{i} / n_{i}=2 \varepsilon_{i} .
$$

It follows from (2.6) that

$$
\left|P\left(x_{k}\right)\right| \leqslant|P(y)|-2 \varepsilon_{i}=1-2 \varepsilon_{i},
$$

and, with (2.5) and $\varepsilon_{i}$ sufficiently small, that

$$
\operatorname{sgn} P\left(x_{k}\right)=\operatorname{sgn}\left(f-T_{n_{i}}(f)\right)\left(x_{k}\right) .
$$

Thus for each $P \in S_{n_{i}}$ we have

$$
\begin{aligned}
\max _{x \in A_{m}(f)}\left\{\operatorname{sgn}\left(f-T_{n_{i}}(f)\right)(x)\right\} P(x) & \geqslant\left\{\operatorname{sgn}\left(f-T_{n_{i}}(f)\right)\left(x_{k}\right)\right\} P\left(x_{k}\right) \\
& =\left|P\left(x_{k}\right)\right| \geqslant 1-2 \varepsilon_{i} .
\end{aligned}
$$

However, it is known [1, p. 81 , proof of the Strong Unicity Theorem] that

$$
M_{n_{i}}^{T}(f)=\left(\min _{P \in S_{n_{i}}} \max _{x \in E_{n_{i}}(f)}\left\{\operatorname{sgn}\left(f-T_{n_{i}}(f)\right)(x)\right\} P(x)\right)^{-1} .
$$

So, noting that $A_{n_{i}}(f) \subset E_{n_{i}}(f)$, we have

$$
M_{n_{i}}^{T}(f) \leqslant\left(1-2 \varepsilon_{i}\right)^{-1}
$$

On the other hand, by (1.1),

$$
\left\|T-T_{n_{i}}(f)\right\| \leqslant M_{n_{i}}^{T}(f)\left(\|f-T\|-\left\|f-T_{n_{i}}(f)\right\|\right)<M_{n_{i}}^{T}(f)\left\|T-T_{n_{i}}(f)\right\|
$$

for any $T \in \Pi_{n}^{T}$. Therefore $M_{n_{i}}^{T}(f) \geqslant 1$. Hence Theorem 1 is proved.

3. Condition for $\varlimsup_{n \rightarrow \infty} M_{n}^{T}(f)=+\infty$.

THEOREM 2. If $f \in C([0,2 \pi])$ is not a trigonometric polynomial and if there exists a strictly increasing sequence $\left\{n_{i}\right\}_{i=1}^{\infty}$ of positive integers such that, for each $n_{i}, E_{n_{i}}(f)$ has exactly $2 n_{i}+2$ points, all in $(0,2 \pi)$, then

$$
\varlimsup_{i \rightarrow \infty} M_{n_{i}}^{T}(f)=+\infty \text {. }
$$


Proof. For each $n$, let $E_{n}(f)=\left\{x_{i}: i=0, \ldots, 2 n+1\right\}$ so that $0<x_{0}<x_{1}$ $<\cdots<x_{2 n+1}<2 \pi$.

We first prove that there is a strictly increasing subsequence $\left\{m_{p}\right\}_{p=1}^{\infty}$ of $\left\{n_{i}\right\}_{i=1}^{\infty}$ such that for each $m_{p}$ there exist a $k_{p} \in\left\{0,1, \ldots, 2 m_{p}+1\right\}$ and a $\mathscr{P}_{m_{p}} \in \Pi_{m_{p}}^{T}$ satisfying

$$
\left|\mathscr{P}_{m_{p}}\left(x_{i}\right)\right| \leqslant 1 \text { for } i=0, \ldots, 2 m_{p}+1 \text { and } i \neq k_{p}
$$

and

$$
\lim _{p \rightarrow \infty}\left(\max _{x \in J}\left|\mathscr{P}_{m_{p}}(x)\right|\right)=+\infty
$$

where $J=\left[x_{k_{p}-1}, x_{k_{p}+1}\right], x_{-1}=0$, and $x_{2 m_{p}+2}=2 \pi$.

For each $n$ and $g \in C[0,2 \pi]$, let $L_{n}$ denote the trigonometric interpolation operator into $\Pi_{n}^{T}\left[4\right.$, p. 365 , (34)] so that $L_{n} g \in \Pi_{n}^{T}$ and $L_{n} g\left(x_{i}\right)=g\left(x_{i}\right)$ for $i=1,2, \ldots, 2 n+1$. By a well-known result in $[1$, p. 212], applicable to the subspace $C_{2 \pi}[0,2 \pi]$, we have

$$
\left\|L_{n}\right\|>\left(4 / \pi^{2}\right) \log n
$$

where $\left\|L_{n}\right\|=\sup _{\|g\|=1}\left\|L_{n} g\right\|$. Then, using a similar argument as in the proof of the lemma in $\left[6\right.$, p. 219], we obtain a subsequence $\left\{m_{p}\right\}_{p=1}^{\infty}$ and trigonometric polynomials $\mathscr{P}_{m_{p}}(x)$ that have the required properties.

Next, for each $m_{p}$, consider the $2 m_{p}+2$ points $\left\{x_{i}\right\}_{i=0}^{2 m_{p}+1}$ of $E_{m_{p}}(f)$ and, by trigonometric interpolation, construct, for each $j, j=0,1, \ldots, 2 m_{p}+1$, the trigonometric polynomials $q_{j}$ such that

$$
q_{j}\left(x_{i}\right)=(-1)^{i}, \quad i=0,1, \ldots, 2 m_{p}+1 \text { and } i \neq j .
$$

We wish to show that

$$
\lim _{p \rightarrow \infty} \max _{0<j<2 m_{p}+1}\left\|q_{j}\right\|=+\infty
$$

Let $k \in\left\{0,1, \ldots, 2 m_{p}+1\right\}$. For each $i, i=0,1, \ldots, 2 m_{p}+1$, and $i \neq k$, consider the trigonometric polynomial

$$
t_{i}(x)=\prod_{\substack{j=0 \\ j \neq i, k}}^{2 m_{p}+1} \frac{\sin \frac{1}{2}\left(x-x_{j}\right)}{\sin \frac{1}{2}\left(x_{i}-x_{j}\right)}
$$

Since $\operatorname{sgn}\left(\sin \frac{1}{2}\left(x-x_{j}\right)\right)=\operatorname{sgn}\left(x-x_{j}\right)$ when $0<\frac{1}{2}\left|x-x_{j}\right|<\pi$, by examining the signs of $x-x_{j}$ and $x_{i}-x_{j}$, it is easy to see that, for $x \in\left(x_{k-1}, x_{k+1}\right)$ where $x_{-1}=0, x_{2 m_{p}+2}=2 \pi$, sgn $t_{i}(x)=(-1)^{k-i-1}$. Hence $\operatorname{sgn}\left\{t_{i}(x)(-1)^{i}\right\}$ have the same value for $x \in\left(x_{k-1}, x_{k+1}\right)$ and $i=0,1, \ldots, 2 m_{p}+1, i \neq k$. Then following an argument similar to that in the proof of Theorem 3 in [6, pp. 219-220], we have

$$
\left\|q_{k_{p}}\right\|>\max _{x \in\left[k_{p}-1, k_{p}+1\right]}\left|\mathscr{P}_{m_{p}}(x)\right|
$$

where $k_{p}$ and $\mathscr{P}_{m_{p}}(x)$ satisfy (3.1). Thus

$$
\lim _{p \rightarrow \infty} \max _{0<j<2 m_{p}+1}\left\|q_{j}\right\|=+\infty \text {. }
$$


Since the dimension of the Haar subspace $\Pi_{m_{p}}^{T}$ is $2 m_{p}+1$, by Theorem 5 and Lemma 3 in [2], we have

$$
M_{m_{p}}^{T}(f)=\max _{0<j<2 m_{p}+1}\left\|q_{j}\right\|
$$

Hence

$$
\lim _{p \rightarrow \infty} M_{m_{p}}^{T}(f)=+\infty
$$

and the theorem is proved.

The above theorem gives a sufficiency condition for $\left\{M_{n}^{T}(f)\right\}_{n=1}^{\infty}$ to be unbounded. The proof remains essentially the same when the extremal points are in $[0,2 \pi)$ instead of $(0,2 \pi)$. However, in this case, the functions in $C_{2 \pi}[0,2 \pi]$ have to be treated as a special case. Here we have given the theorem in the more simple form.

\section{An example.}

THEOREM 3. For any positive integer $m$, there exist a periodic function $f$, with period $2 \pi$ and continuous derivatives up to order $m$, and an increasing sequence $\left\{n_{i}\right\}_{i=1}^{\infty}$ of positive integers such that

$$
\lim _{i \rightarrow \infty} M_{n_{i}}^{T}(f)=1 \text { and } \lim _{i \rightarrow \infty} M_{n_{i}-1}^{T}(f)=+\infty .
$$

We see that $M_{n}^{T}(f)$ may change its value rapidly at two adjacent values of $n$ as $n \rightarrow \infty$.

ProOF. We prove the theorem by constructing the periodic function $f$ explicitly.

Let $\delta=\sqrt{2} \pi$. Define a function $f$ on $[0,2 \pi]$ by

$$
f(x)=\sum_{k=0}^{\infty} b_{k} \cos a_{k}(x-\delta),
$$

where $a_{k}=3^{k^{2}}$ and $b_{k}=2^{-k} a_{k}^{-m}$, with $m$ an arbitrary given positive integer. By the Weierstrass $M$-test, one can easily verify that $f$ has continuous derivatives up to order $m$. Let

$$
\mathcal{T}_{a_{n}}(x)=\sum_{k=0}^{n} b_{k} \cos a_{k}(x-\delta) .
$$

Then

$$
\left(f-\mathcal{T}_{a_{n}}\right)(x)=\sum_{k=n+1}^{\infty} b_{k} \cos a_{k}(x-\delta) .
$$

If $k>n+1$, the numbers $a_{k} / a_{n+1}$ are always odd integers and $f-\mathcal{T}_{a_{n}}$ attains its maxima and minima, which are of equal magnitude $\sum_{k=n+1}^{\infty} b_{k}$, alternately at the points, and only at the points,

$$
\xi_{j}=\left(\sqrt{2}+j / a_{n+1}\right) \pi,
$$

where $j$ are integers for which $\xi_{j} \in[0,2 \pi]$. The $\xi_{j}$ are equally spaced at a distance $\pi / a_{n+1}$ apart. None of them coincides with an end point of $[0,2 \pi]$, since, for any integer $j, \sqrt{2}+j / a_{n+1}$ is irrational. Hence there are exactly $2 a_{n+1}$ points $\xi_{j}$ in the 
interval $(0,2 \pi)$ of length $2 \pi$. It follows from the alternation theorem that $\mathcal{T}_{a_{n}}$ is the best approximation to $f$ from $\Pi_{a_{n}}^{T}$ and hence

$$
E_{a_{n}}(f)=\left\{\xi_{j} \in[0,2 \pi]: \xi_{j}=\delta+j \pi / a_{n+1} \text { and } j \text { are integers }\right\},
$$

which consists of exactly $2 a_{n+1}$ points. Define $p_{n}$ by $2 p_{n}+2=2 a_{n+1}$, that is

$$
p_{n}=a_{n+1}-1=3^{(n+1)^{2}}-1>3^{n^{2}}=a_{n} \text {. }
$$

With $p_{n}>a_{n}$, it is easy to see that $E_{p_{n}}(f)=E_{a_{n}}(f)$. In other words, there exists a strictly increasing sequence $\left\{p_{n}\right\}_{n=1}^{\infty}$ of positive integers such that $E_{p_{n}}(f)$ has exactly $2 p_{n}+2=2 a_{n+1}$ points. Then by Theorem 2 , there exists a strictly increasing subsequence $\left\{p_{n_{i}}\right\}_{i=1}^{\infty}$ of $\left\{p_{n}\right\}_{n=1}^{\infty}$ such that

$$
\lim _{i \rightarrow \infty} M_{p_{n_{i}}}(f)=+\infty \text {. }
$$

Also, $E_{a_{n}}(f) \cup\{2 \pi, 0\}$ satisfies (2.2) since

$$
\left|\xi_{j}-\xi_{j+1}\right|=\pi / a_{n+1}=\pi\left(3^{-2 n-1}\right) / 3^{n^{2}}=\varepsilon_{n} / a_{n} .
$$

By Theorem 1 we have $\lim _{n \rightarrow \infty} M_{a_{n}}^{T}(f)=1$ where $a_{n}=p_{n-1}+1$. Thus $f$ is the required function and the theorem is proved.

\section{REFERENCES}

1. E. W. Cheney, Introduction to approximation theory, McGraw-Hill, New York, 1966.

2. A. K. Cline, Lipschitz conditions on uniform approximation operators, J. Approx. Theory 8 (1973), 160-172.

3. M. S. Henry and J. A. Roulier, Lipschitz and strong unicity constants for changing dimension, J. Approx. Theory 22 (1978), 85-94.

4. I. P. Natanson, Konstruktive Funktionentheorie, Akademie-Verlag, Berlin, 1955.

5. S. J. Poreda, Counterexamples in best approximation, Proc. Amer. Math. Soc. 56 (1976), 167-171.

6. D. Schmidt, On an unboundedness conjecture for strong unicity constants, J. Approx. Theory 24 (1978), 216-223.

7. W. Wolibner, Sur un polynôme d'interpolation, Colloq. Math. 2 (1951), 136-137.

Department of Mathematics, University of Hong Kong, Hong Kong 